\title{
Birth and decay of coherent optical phonons in femtosecond-laser-excited bismuth
}

\author{
T. Garl, ${ }^{1}$ E. G. Gamaly, ${ }^{2, *}$ D. Boschetto, ${ }^{1}$ A. V. Rode, ${ }^{1}$ B. Luther-Davies, ${ }^{2}$ and A. Rousse ${ }^{1}$ \\ ${ }^{1}$ Laboratoire d'Optique Appliquée, ENSTA/Ecole Polytechnique, Chemin de la Huniere, 91761 Palaiseau Cedex, France \\ ${ }^{2}$ Laser Physics Centre, Research School of Physical Sciences and Engineering, The Australian National University, Canberra, \\ Australian Capital Territory 0200, Australia
}

(Received 13 June 2008; revised manuscript received 10 September 2008; published 8 October 2008)

\begin{abstract}
The transient reflectivity of bismuth crystal excited by a 45 fs laser pulse in the near-infrared range has been recovered with an accuracy of $10^{-5}$, at initial sample temperatures ranging from 50 to $510 \mathrm{~K}$, and at pump fluences from $2 \mathrm{~mJ} / \mathrm{cm}^{2}$ to $21 \mathrm{~mJ} / \mathrm{cm}^{2}$. The coherent phonon excitation and decay processes were imprinted into the time-dependent reflectivity and this allows us to uncover the temporal phonon history preceding the structural transformation of solid Bi. Analysis showed that the first coherent atomic displacement was produced by the polarization force and the electron pressure force during the laser pulse, and that manifests itself by a negative change in the reflectivity. The frequency of the subsequent reflectivity oscillations was chirped, redshifted from the initial value due to the lattice heating. The amplitude decreased gradually while electrons transferred their energy to the lattice. Heating and thermal expansion of the lattice transformed the initially coherent harmonic vibrations of atoms into strongly nonlinear chaotic motion that signifies the onset of disordering of the solid. This process was identified through measurement of the damping rate of the reflectivity oscillations and interpretation of this rate as the decay rate of an optical phonon into two acoustic phonons. The analysis of the reflectivity oscillations provides evidence that the overheated solid experiences only the onset of the solid-liquid phase transition but did not proceed into the liquid phase. General relations between the laser-exerted forces, the atomic motion, and the optical parameters were established. The proposed theory reproduces well the measured transient reflectivity across a wide range of crystal temperatures and laser excitation fluences.
\end{abstract}

DOI: 10.1103/PhysRevB.78.134302

PACS number(s): 78.47.-p, 63.20.-e, 78.68.+m

\section{INTRODUCTION}

With the advent of femtosecond lasers in the early 1980s, time-resolved experiments on a great variety of ultrafast phenomena have become possible. ${ }^{1-23}$ One major focus of this field of research is the excitation and detection of coherent lattice vibrations, which has been realized in many transparent and opaque materials, among which are semimetals, ${ }^{2,3,5-14}$ transition metals, ${ }^{22}$ cuprates,${ }^{23}$ insulators,${ }^{20}$ and semiconductors. ${ }^{1-4,19,21}$ The generation of coherent optical phonons allows deep insight into the microscopic nature of laser-controlled atomic motion. The ability to drive and control coherent lattice vibration via an external photon flux opens a number of interesting applications such as the possibility to induce particular phase transitions (nonthermal melting, ${ }^{1}$ paraelectric-to-ferroelectric ${ }^{16}$ or insulator-to-metal transitions ${ }^{18}$ ), the selective opening of the "caps" of nanotubes in nonequilibrium conditions ${ }^{17}$ or providing a basis for SASER (sound amplification by stimulated emission of radiation $)^{19}$ experiments.

In order to explain the generation of coherent phonons, a number of physical mechanisms have been proposed, for example, displacive excitation of coherent phonons (DECP), ${ }^{2,3}$ which was originally introduced to describe reflectivity oscillations induced by fully symmetric coherent phonons in opaque media, and transient stimulated Raman scattering (TSRS), ${ }^{4,5}$ which is applicable to both opaque and transparent media. Despite the popularity of these theories and their ability to account for certain experimental findings, a theory which can explain the entirety of experimental results concerning the generation of coherent phonons in transparent and opaque media and their connection to transient reflectivity changes is, to the best of our knowledge, still absent. In particular, novel effects, like a sharp drop in reflectivity ${ }^{24}$ at time scales of less than $40 \mathrm{fs}$ and a negative change in reflectivity after electron-lattice equilibration in bismuth, have remained obscure.

In what follows, we present a detailed experimental and theoretical study of reflectivity oscillations recorded in optically excited bismuth over a time span of $30 \mathrm{ps}$ with a resolution of 35 fs taking into account the dependence on initial sample temperature over the range from 50 to $510 \mathrm{~K}$, and excitation fluence from 2 to $21 \mathrm{~mJ} / \mathrm{cm}^{2}$. From the measurements, the subtleties of ultrafast atomic motion have been recovered. These include first a rapid atomic displacement followed by excitation and decay of vibrational modes, whose life is compared with times for nonlinear phononphonon interactions, the disappearance of harmonic vibrations, and finally the onset of thermal expansion and instability resulting in a transient state described neither by a solid nor a liquid phase. The interconnected processes of electron heating by laser absorption, the interaction within and between the electronic and lattice subsystems, the forces driving the atomic motion as well as the changes in optical properties induced by these effects have been considered in succession.

The paper is structured as follows: In Sec. II the details of the experiments are outlined and the experimental results are presented. In Sec. III, the elements of our theoretical approach are described. The time scales for electron-electron, electron-phonon, and phonon-phonon interaction are compared and the expressions for the excitation of electrons and the laser-exerted forces on the material are derived. The fast 
atomic displacement observed before the onset of coherent vibrations and the coherent vibrational atomic displacements have been considered. Nonlinear phenomena that appeared in the process of energy transfer from the electrons to the lattice have been taken into account in calculations of transient reflectivity. In Sec. IV, the results are discussed and Sec. V presents a summary and a conclusion to our results.

\section{EXPERIMENT}

\section{A. Experimental technique and setup}

Time-resolved reflectivity measurements have been carried out using a single crystal of bismuth cut with (111) orientation with respect to the trigonal axis representation; standard x-ray diffraction analysis has shown a good crystalline quality of the sample. Laser pulses with a duration of 40-45 fs delivered by a Ti:sapphire laser system pumped by the second harmonic of a Nd:YAG laser at a repetition rate of $1 \mathrm{KHz}$ were applied in standard pump-probe geometry. The $s$-polarized probe pulses were focused to a $40 \mu \mathrm{m}$ spot and carefully superposed with the center of the $p$-polarized pump pulses with a diameter of $125 \mu \mathrm{m}$ in order to ensure probing of a preferably homogenously excited region of the sample. The spatial overlap and the beam dimensions were monitored using a charge coupled device (CCD) camera with a microscope imaging system. Crossed polarizations for pump and probe were used in order to reduce the influence of scattered pump light with the help of a polarizer placed before the photodiode detecting the transient reflectivity signal. The angle of incidence of the probe beam was approximately $10^{\circ}$ to the normal angle, while the pump was normal to the target surface.

To allow lock-in detection, the pump beam was chopped at $500 \mathrm{~Hz}$ using a phase-locked system ensuring perfect synchronization of the chopper and the laser pulse by real-time control of rotation speed and phase. By using a reference beam, intensity fluctuations of the laser were taken into account. With an integration time of $1 \mathrm{~s}$ the detection of reflectivity changes with an accuracy of $\Delta R / R_{0} \sim 10^{-5}$ was achieved, $R_{0}$ denoting unperturbed reflectivity before the arrival of the pump pulse.

The sample was heated with a resistance cartridge heater for measurements between the room temperature and $510 \mathrm{~K}$, and cooled in a closed-cycle cryostat for measurements between the room temperature and $50 \mathrm{~K}$; the sample temperature was measured using a thermocouple. The equivalence of the different series of experiments was ensured by precise agreement between the measurements at room temperature for each series.

\section{B. Temperature and fluence dependencies of transient reflectivity}

The reflectivity signal for an excitation with pump fluence of $6.9 \mathrm{~mJ} / \mathrm{cm}^{2}$ for five different temperatures is shown in Fig. 1. The behavior of the time-dependent reflectivity can be described by a superposition of an oscillatory and a nonoscillatory component. Due to the excitation by the pump pulse, the reflectivity increases to a maximum reached at about 200

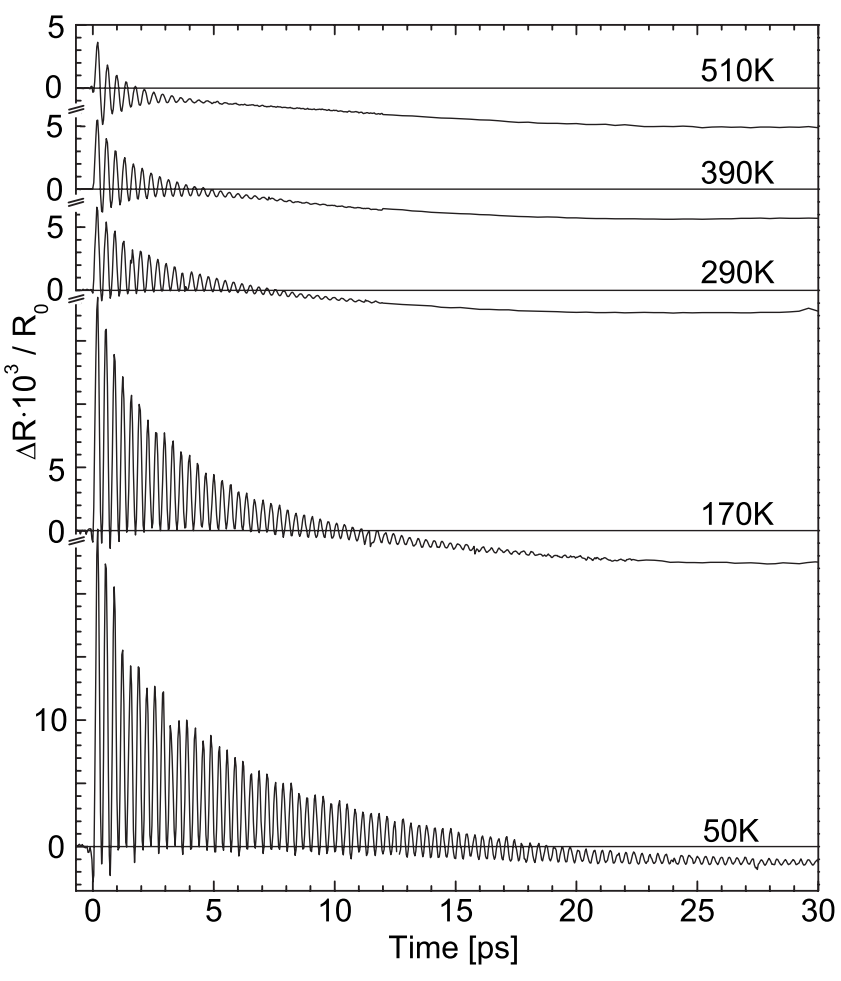

FIG. 1. Transient reflectivity signals $\Delta R / R_{0}$ for different crystal temperatures at a constant pump fluence of $6.9 \mathrm{~mJ} / \mathrm{cm}^{2}$. The curves at temperatures higher than $50 \mathrm{~K}$ are offset for better readability; the horizontal lines indicate the zero level for each depicted timedomain signal.

fs and then starts to oscillate with a temperature-dependent frequency while the amplitude of oscillation decays nearly exponentially with time. The oscillations phase out with a temperature-dependent delay ranging from a few ps for 510 $\mathrm{K}$ to $35 \mathrm{ps}$ for $50 \mathrm{~K}$. The reflectivity drops below the unperturbed value and reaches a minimum before slowly returning to the unperturbed value after $\sim 4$ ns. The time at which the sign change of $\Delta R / R_{0}$ occurred, the time when the minimum was reached, and the absolute value of $\Delta R / R_{0}$ at the minimum were all temperature dependent. In our previous experiments, a sharp initial drop of reflectivity was observed. ${ }^{24}$ Here this feature was less pronounced due to the slightly longer pulse duration used in the current experiments. At 50 $\mathrm{K}$, the reflectivity signal showed a superposition of two oscillations at different frequencies. A Fourier analysis showed that a frequency component corresponding to the nonsymmetric $E_{g}$ mode of $2.12 \mathrm{THz}$ had appeared in addition to the $A_{1 g}$ mode. The observation of this mode as well as its absence at higher temperatures is in agreement with earlier findings. ${ }^{7}$

Figure 2 shows the frequency of the $A_{1 g}$ mode as a function of crystal temperature. Since the frequency also varies with delay between the pump and the probe pulse, we determined the initial frequency by fitting a damped harmonic oscillation superimposed to an exponential decay to the first two oscillations. The error bars in the plot result from the fit. As the temperature increases, the frequency is shifted to lower values. Figure 3 depicts the temperature dependence of the damping of the oscillations, estimated by supposing an 


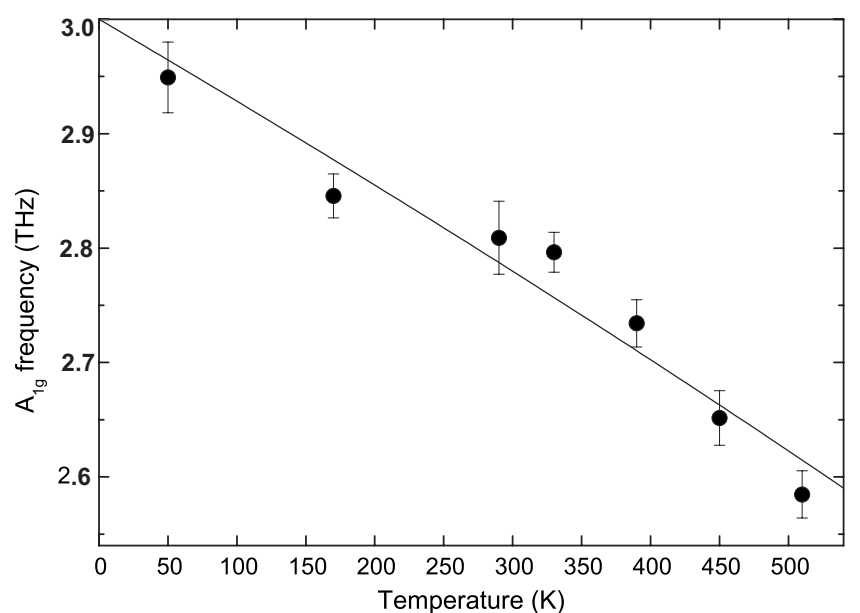

FIG. 2. Initial frequency of the $A_{1 g}$-phonon mode as a function of the crystal temperature at a constant pump fluence of $6.9 \mathrm{~mJ} / \mathrm{cm}^{2}$; the line corresponds to a fit performed with Eq. (39) — see Sec. IV in the text below.

exponential decay of the oscillation amplitude.

At room temperature, excitation-fluence-dependent measurements have been carried out and the corresponding reflectivity changes are depicted in Fig. 4. The characteristics of the time-domain signals have changed dramatically with the fluence. Concerning the oscillatory component, the redshift of the frequency increased linearly with pump fluence (see Fig. 5), which has been shown to be valid for the fluences up to $8 \mathrm{~mJ} / \mathrm{cm}^{2}{ }^{25}$ The damping rate of the phonon linearly increased with fluence, as shown in Fig. 6.

\section{THEORY}

The experimental results on time-dependent reflectivity measurements with high accuracy contain many subtle features. Our goal here is to trace the atomic motion and the

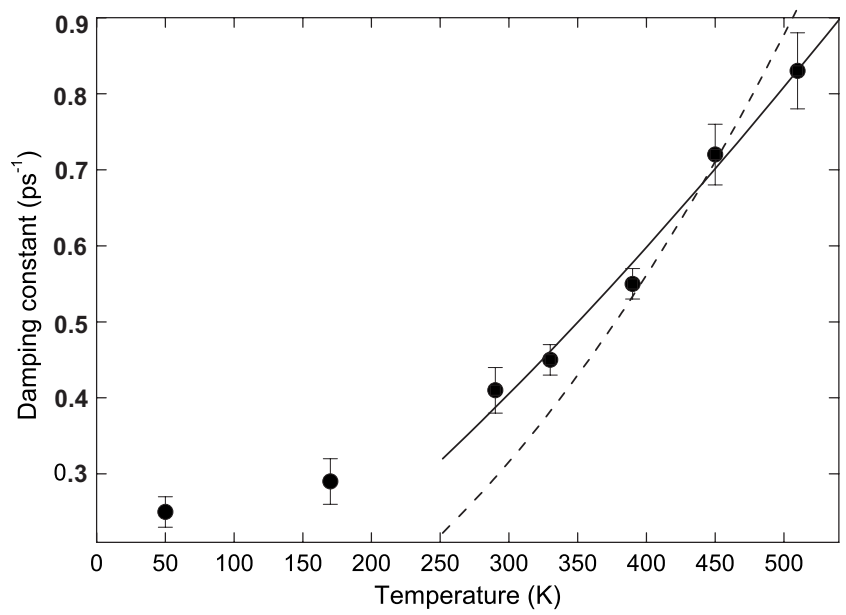

FIG. 3. Damping constant of the $A_{1 g}$ mode as a function of crystal temperature at a constant fluency of $6.9 \mathrm{~mJ} / \mathrm{cm}^{2}$. The dashed line corresponds to a fit with Eq. (38), and the solid line to a fit with Eq. (38) where the exponent was a fitting parameter-see Sec. IV in the text below.

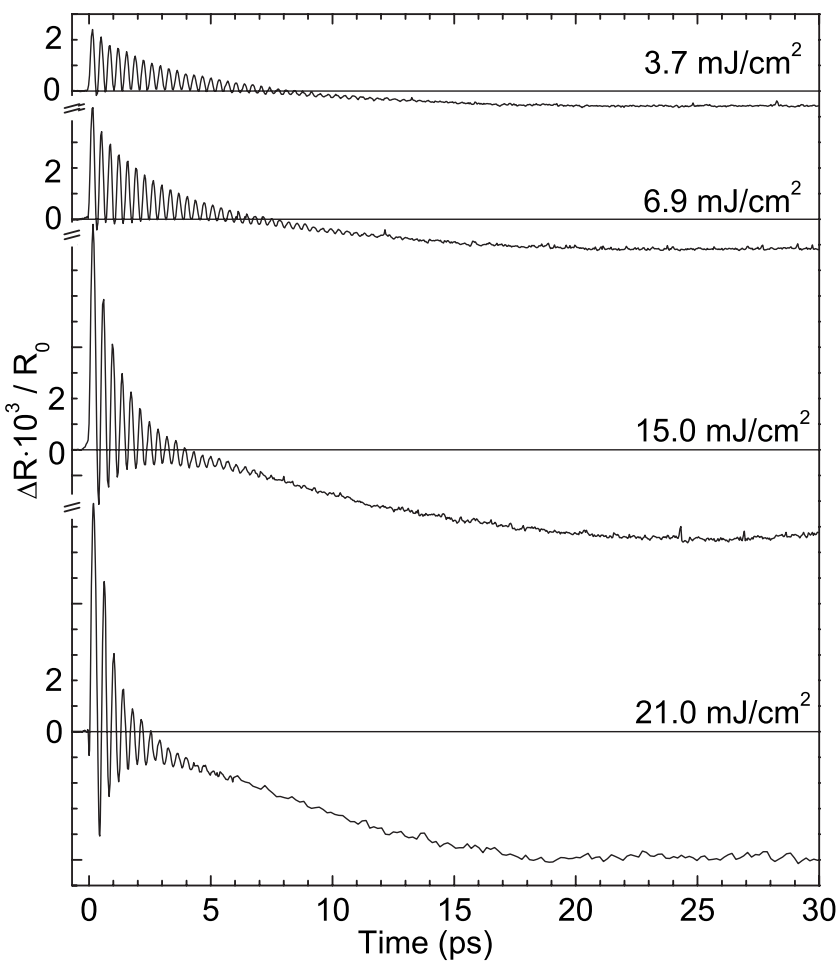

FIG. 4. Transient reflectivity signals $\Delta R / R_{0}$ for various pump fluences at room temperature. The horizontal lines correspond to the zero level for each depicted time-domain signal.

subsequent phase changes by the time-dependent dielectric properties of laser-affected material. In the following theoretical analysis we established the general relations between the laser excitation and the laser-exerted forces, which cause the atomic vibrations with the phonon frequency at the initial crystal temperature. We next demonstrate how the atomic vibrations affect the optical properties through the electronphonon interactions. The nonlinear multiphonon processes are responsible for the gradual decay of phonon oscillations and for the onset of the material transformation, which are imprinted into the time-dependent reflectivity. This analysis

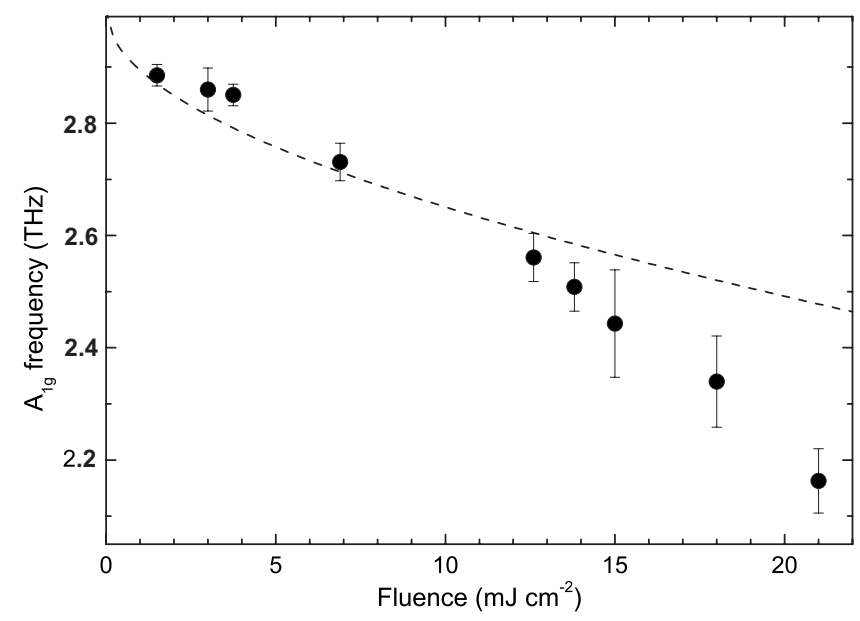

FIG. 5. Initial frequency of the $A_{1 g}$ mode as a function of the pump fluence at room temperature; the dashed line corresponds to frequencies calculated with Eq. (40)_-see Sec. IV in the text below. 


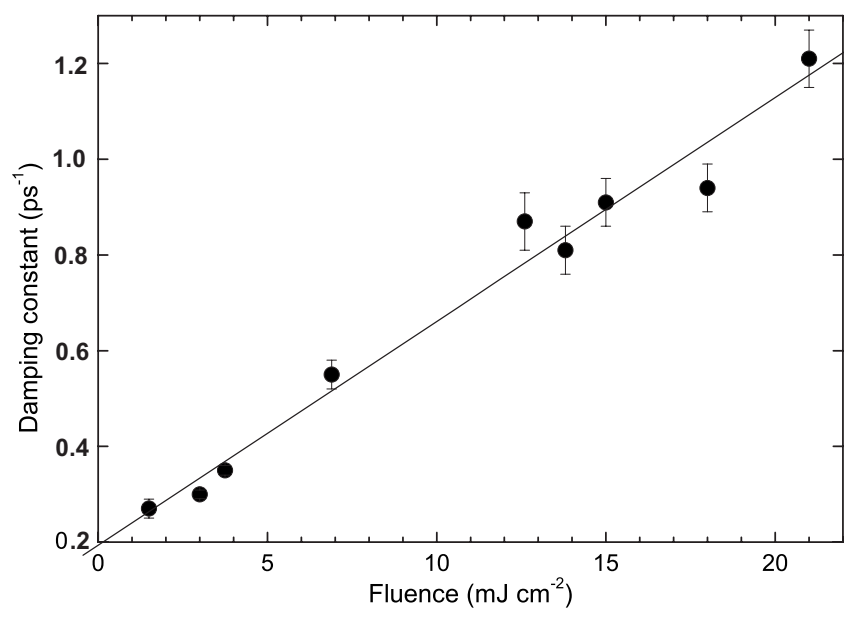

FIG. 6. Damping constant of the $A_{1 g}$ mode as a function of pump fluence at room temperature; the line corresponds to a linear fit to the data points.

allowed us to uncover, through the temporal phonon history, the structural transformation of solid $\mathrm{Bi}$ under fs-laser excitation.

\section{A. Transient properties of a laser-excited solid}

\section{Relaxation times: quasiequilibrium electron and lattice temperatures}

Electron-electron collisions quickly lead to the establishment of a local equilibrium energy distribution. The electronelectron collision rate for a strongly correlated degenerate electron gas has a form: ${ }^{26} \nu_{e e} \propto \omega_{p} \varepsilon_{e} / \varepsilon_{F}$ ( $\varepsilon_{e}$ is the electron energy in excess over the Fermi level, $\varepsilon_{F}$, and $\omega_{p}$ is plasma frequency). For the conditions of the experiment, $\varepsilon_{e}$ $\cong 0.02 \varepsilon_{F}$, and $\omega_{p e}=1.3 \times 10^{16} \mathrm{~s}^{-1}\left(\omega_{p e}\right.$, and $\varepsilon_{F}$, are extracted from the optical data). ${ }^{27-29}$ Thus an equilibrium distribution for electrons is established in a period, $t_{e e} \approx v_{e e}^{-1} \sim 4$ fs.

Similarly phonon-phonon interactions lead to the establishment of an equilibrium in the phonon subsystem (lattice temperature). The effective phonon-phonon collision rate reads $^{30,31} \nu_{\text {ph-ph }} \cong \omega_{D} T_{L} / T_{D}\left(\omega_{D}, T_{D}\right.$, and $T_{L}$ are, respectively, the Debye frequency, the Debye temperature, and lattice temperature). The phonon-phonon relaxation time in our case $\left(\omega_{D}=1.56 \times 10^{13} \mathrm{~s}^{-1} ; T_{D}=119 \mathrm{~K}\right)$ lies in the range $t_{\mathrm{ph}-\mathrm{ph}}$ $=\left(n_{\mathrm{ph}-\mathrm{ph}}\right)^{-1} \sim 8-80$ fs.

The transfer of energy from the electrons to phonons heats the lattice. The heating time is determined by the electronphonon energy transfer rate: ${ }^{26,30,31} t_{e-\mathrm{ph}}^{\text {energy }}=\left(\nu_{e \text {-ph }}^{\text {energy }}\right)^{-1}$ $\approx\left(\hbar \omega_{D}^{2} T_{L}^{2} / T_{D}^{2} \varepsilon_{F}\right)^{-1}$. In the conditions of our experiments $t_{e \text {-ph }}^{\text {energy }}$ varies in the range 5-20 ps.

Heat diffusion appears to be less important compared with other relaxation processes and hence it is neglected in the following. In fact, the time for cooling the skin depth of $l_{s}$ $=29.8 \mathrm{~nm}$ is $t_{\text {cool }} \approx l_{s}^{2} / D=119 \mathrm{ps}$ (the diffusion coefficient in Bi $\left.D=0.067 \mathrm{~cm}^{2} / \mathrm{s}\right)^{28}$ and this should be compared to the observation period $(\sim 25 \mathrm{ps})$ in our experiments. Thus the main nonequilibrium process is electron-phonon temperature equilibration while electron-electron and phonon-phonon equilibration adiabatically follow to the slower evolution of the lattice heating. The above relation between relaxation times is the rationale for using the 2-temperature approximation $\left[T_{e}(t), T_{L}(t)\right]$ to describe the nonequilibrium laser-matter interaction. ${ }^{26,32}$

\section{Electron and lattice temperature in $\mathbf{2} \mathbf{T}$ approximation}

We obtained $T_{e}(t), T_{L}(t)$ from numerical solutions of coupled electron and lattice energy equations ${ }^{26,32}$ with material data taken from Refs. 27-29. The maximum electron temperature at the end of the pulse is found to be $T_{e, \max }$ $=\left[4 \varepsilon_{F} A F\left(t_{p}\right) / \pi^{2} n_{e} l_{s}\right]^{1 / 2}$; here $A=1-R=0.26$ is the absorption coefficient, $F\left(t_{p}\right)$ is the laser fluence, $t_{p}$ is the laser pulse duration, and $n_{e}$ is the electron density. The maximum lattice temperature is reached after equilibration of electron and lattice temperatures after $\sim 20 \mathrm{ps}$, and is, for example, $701.5 \mathrm{~K}$ and $1273 \mathrm{~K}$ for the fluences $2.7 \mathrm{~mJ} / \mathrm{cm}^{2}$ and $6.7 \mathrm{~mJ} / \mathrm{cm}^{2}$ correspondingly. Note that in both cases the lattice temperature is higher than the equilibrium melting point $(544.6 \mathrm{~K})$ for $\mathrm{Bi}: 1.29$ and 2.34 times, respectively. From such considerations we can obtain the time dependence of the electron number density, and electron and lattice temperatures, which allow calculation of the transient dielectric function.

\section{Electron excitation from valence to conduction band}

The number density of electrons excited to the conduction band by an avalanche-like process is proportional to the electron energy density: $n_{e}\left(t_{p}\right) \cong \frac{2 A F\left(t_{p}\right)}{\Delta E l_{s}}$ (here $\Delta E$ is the energy required for a transition from the valence to the conduction band, which is $\sim 0.1 \mathrm{eV}$ ). The electron number density at the end of the pulse is proportional to the electron temperature. After the end of the pulse the number of excited electrons decreases due to recombination. Because the recombination rate for $\mathrm{Bi}$ is unknown to the best of our knowledge, we assume that in the cooling stage after the pulse the above proportionality also holds, $\Delta n_{e} / n_{e} \propto T_{e}(t) / T_{e, \max }$, where $T_{e, \max }$ is the maximum electron temperature at the end of the pump pulse.

\section{Absorbed energy density and stress tensor}

The laser pulse is shorter than the electron-phonon energy transfer time. Therefore only electrons are heated during the pulse. The electron energy density absorbed in the skin layer, $E_{e l}=C_{e} n_{e} T_{e}=2 A F\left(t_{p}\right) / l_{s}$ reaches a maximum at the end of the pulse; here $C_{e}$ is heat capacity, $T_{e}$ is the electron temperature, and $l_{s}=2.984 \times 10^{-6} \mathrm{~cm}$ is the skin depth. ${ }^{33}$ For example, the absorbed energy density for $2.7 \mathrm{~mJ} / \mathrm{cm}^{2}$ and $6.7 \mathrm{~mJ} / \mathrm{cm}^{2}$ equals to $0.48 \times 10^{3} \mathrm{~J} / \mathrm{cm}^{3}$ and 1.19 $\times 10^{3} \mathrm{~J} / \mathrm{cm}^{3}$ correspondingly, and it should be compared to the enthalpy of melting in equilibrium of $0.5 \mathrm{~kJ} / \mathrm{cm}^{3}{ }^{28}$

Accordingly, after the electron-electron and phononphonon equilibration one can use the equilibrium concept of the stress tensor in a laser-affected solid in the same form as in equilibrium conditions. For an initially isotropic medium the stress tensor reads ${ }^{34}$

$$
\sigma_{i k}=-P \cdot \delta_{i k}-\frac{E^{2} \varepsilon \cdot \delta_{i k}}{8 \pi}+\frac{E^{2}}{8 \pi}\left[n_{a}\left(\frac{\partial \varepsilon_{j k}}{\partial n_{a}}\right)_{T}\right] \delta_{i j}+\frac{E_{i} D_{k}}{4 \pi},
$$

where the electric field displacement vector has the form $D_{k}=\varepsilon_{k j} E_{j}$. We assume that the dielectric tensor modified by 
the laser effect consists of two terms, the Drude-type term, $\varepsilon_{D}$, and the polarization term, $\varepsilon_{j k}^{(p)}$ :

$$
\varepsilon_{j k}=\varepsilon_{D} \cdot \delta_{j k}+\varepsilon_{j k}^{(p)} .
$$

The difference between the equilibrium state and the transient state is that in equilibrium $n_{e}$ is constant and the electron temperature is equal to the lattice temperature, while in the transient sate $n_{e}$ is changing and the electron temperature is much higher than that of the lattice. The pressure in the transient state is the sum of the contributions from the laseraffected electron and lattice subsystems, $P=P_{e}+P_{L}$, and the dielectric function is modified by the laser action.

\section{B. Forces imposed by the laser pulse on a solid}

\section{Laser-exerted forces driving atomic motion}

The action of an external electric field on a solid (liquid) at moderate intensity leads to the internal deformations (atomic motion) while the total volume remains constant. These deformations are accompanied by a change in the initially homogeneous dielectric function. The volume force induced by the laser field is expressed through the stress tensor, $\sigma_{i k}$, and modified by the field action, were found as the following: ${ }^{34}$

$$
\begin{aligned}
f_{i}=\frac{\partial \sigma_{i k}}{\partial x_{k}} & =-\frac{\partial P}{\partial x_{i}}-\frac{\partial \varepsilon_{i k}^{(p)}}{\partial x_{k}} \cdot \frac{E^{2}}{8 \pi}+\frac{\left(\varepsilon_{D}-1\right)}{8 \pi} \frac{\partial E^{2}}{\partial x_{i}} \\
& =f_{i}^{\text {th }}+f_{i}^{(p)}+f_{i}^{\text {pond }} .
\end{aligned}
$$

Here we took into account that for the Drude-type function the following relation holds: $n_{a}\left(\partial \varepsilon_{D} / \partial n_{a}\right)_{T}=\varepsilon_{D}-1$. The first term in Eq. (3) is the thermal force. During the pulse when only the electrons are excited while the lattice is cold, the gradient of electronic pressure is a manifestation of the electrostatic interaction between the electrons and the ions. The second term is the polarization force when the polarizability depends on the atomic displacement (the Placzek effect), ${ }^{35}$ and the third term is the ponderomotive force. Note that polarization and ponderomotive forces are effective only during the pulse, while the thermal force drives atomic motion until the spatial smoothing of temperature gradients. It is also worth noting that the polarization force in Eq. (3) is similar but not identical to the force driving phonon excitation in the Raman effect. ${ }^{35}$ With a laser pulse duration much shorter than the phonon period there is no time for this force to oscillate with the phonon's frequency.

Let us compare the laser-exerted forces to each other. We take the polarizability (and dielectric) tensor in the Placzek form, $\chi_{i k}=\chi_{0, i k}+\left(\frac{\partial \chi_{i k}}{\partial x_{l}}\right)_{0} \cdot x_{l} \cdot{ }^{35}$ Then the polarization term in Eq. (2) reads

$$
\varepsilon_{i k}^{(p)}=1+4 \pi \chi_{0, i k}+4 \pi \cdot\left(\frac{\partial \chi_{i k}}{\partial x_{l}}\right)_{0} \cdot x_{l}
$$

where $x_{l}$ is the atomic displacement in $l$ direction. Then the polarization force in Eq. (3) reduces to the following:

$$
f_{i}^{(p)}=\frac{\partial \varepsilon_{i k}^{(p)}}{\partial x_{k}} \cdot \frac{E^{2}}{8 \pi}=\left(\frac{\partial \chi_{i k}}{\partial x_{k}}\right)_{0} \cdot \frac{E^{2}}{2} .
$$

We assume that $\left(\partial \chi_{i k} / \partial x_{k}\right)_{0} \approx \chi_{0} / d, d$ being the interatomic distance. We calculate the unperturbed polarizability with the Lorentz-Lorenz formula, $\chi_{0}=3(\varepsilon-1) / 4 \pi(\varepsilon+2) .{ }^{36}$ The contribution of all terms in Eq. (3) to the end of the laser pulse can be estimated as follows:

$$
f^{\text {th }} \approx \frac{n_{e} T_{e}}{l_{s}}, \quad f^{(p)} \approx \frac{4 \pi \cdot \chi_{0}}{d} \frac{I}{c}, \quad f \text { pond } \approx \frac{\left(\varepsilon_{D}-1\right)}{l_{s}} \frac{I}{c} .
$$

Here, the average laser intensity during the pulse, I $=c E^{2} / 8 \pi$, was introduced. For example, in bismuth $\left(\varepsilon_{D}\right.$ $=22.39$ at $800 \mathrm{~nm} ; d=3.3 \times 10^{-8} \mathrm{~cm} ; n_{a}=2.82 \times 10^{22} \mathrm{~cm}^{-3}$ $n_{e}=5.34 \times 10^{22} \mathrm{~cm}^{-3}$ ) one obtains for $\chi=0.2 ; \chi_{0} / d \approx 6.34$ $\times 10^{6} \mathrm{~cm}^{-1}$. In the conditions of the experiments $\left(t_{p}=40 \mathrm{fs}\right.$; $l_{s}=29.8 \mathrm{~nm}$; fluence $6.9 \mathrm{~mJ} / \mathrm{cm}^{2} ; \quad I=1.75 \times 10^{11} \mathrm{~W} / \mathrm{cm}^{2}$; $I / c=0.58 \times 10^{8} \mathrm{erg} / \mathrm{cm}^{3} ; T_{e}=0.43 \mathrm{eV}$ ) these forces at the end of the pulse are the following:

$$
\begin{aligned}
f^{\text {th }} & \approx 1.3 \times 10^{16} \mathrm{erg} / \mathrm{cm}^{4}>f^{(p)} \\
& \approx 4.61 \times 10^{15} \mathrm{erg} / \mathrm{cm}^{4}>f^{\text {pond }} \\
& \approx 4.38 \times 10^{14} \mathrm{erg} / \mathrm{cm}^{4} .
\end{aligned}
$$

Thus, the thermal force dominates at the end of the pulse. However, one has to take into account that the thermal force goes up almost linearly in time during the pulse. For example, for the top-hat temporal intensity distribution the polarization and ponderomotive forces dominate during the first $10 \%$ of the pulse. Then the thermal force becomes larger and finally it is 2 orders of magnitude larger than the other forces. The forces above are the volume forces. One has to convert them into the forces acting on a single atom by dividing the above results by the atomic number density. Finally,

$$
\begin{aligned}
F_{\mathrm{th}}=f^{\mathrm{th} / n_{a}} & \approx 46 \times 10^{-8} \mathrm{erg} / \mathrm{cm}>F^{(p)} \\
& \approx 16.33 \times 10^{-8} \mathrm{erg} / \mathrm{cm}>f \text { pond } \\
& \approx 1.55 \times 10^{-8} \mathrm{erg} / \mathrm{cm} .
\end{aligned}
$$

\section{Equation of atomic motion in laser-excited solid}

We are going to consider the swift excitation of atomic vibrations by the force acting during a time much shorter than the phonon period in an elemental solid with identical atoms of mass $M$. For a description of strong excitation the spatial dispersion can be neglected and the excited phonon can be considered as a standing wave. The phonon frequency is expressed through the first (quadratic) perturbation term in the interatomic potential, which has the form

$$
\Delta U_{e l}=\frac{1}{2}\left(\frac{\partial^{2} U}{\partial q^{2}}\right)_{0} q^{2} \approx \varepsilon_{b} q^{2} / 2 d^{2} \equiv C q^{2}=M \omega_{0}^{2} q^{2} / 2,
$$

where $q$ is the cold phonon amplitude and $\varepsilon_{b}$ is the binding (cohesive) energy. One can see that the cold phonon frequency derived from Eq. (6) reads $\omega_{0}^{2}=\varepsilon_{b} / M d^{2}$. The elastic force driving atomic vibrations can be expressed through the perturbation of the potential and through the cold phonon amplitude as follows: 


$$
F_{e l}=\frac{\partial \Delta U_{e l}}{\partial q} \approx \varepsilon_{b} q / d^{2}=M \omega_{0}^{2} q .
$$

The magnitude of the cold phonon amplitude (at a temperature lower than the Debye temperature) can be estimated on the basis of the adiabaticity principle ${ }^{31}$ as the following:

$$
q_{0} \approx\left(\frac{\hbar}{M \omega_{0}}\right)^{1 / 2} .
$$

In the unexcited $\mathrm{Bi}$ the longitudinal $A_{1 g}$ optical mode (vibration in $c$ direction) has a frequency $3 \times 10^{12} \mathrm{~s}^{-1}$. An estimation of the phonon frequency via the perturbation in the interatomic potential taking the corresponding values for bismuth $\left(M_{\mathrm{Bi}}=3.47 \times 10^{-22} \mathrm{~g}\right.$, interatomic distance in $c$ direction $d=3.3 \AA$, and $\varepsilon_{b}=2.16 \mathrm{eV}$ ) gives the correct value for the phonon frequency. Calculating the amplitude of the cold phonon one obtains $q_{0}=10^{-9} \mathrm{~cm}$; the elastic force equals $F_{e l}=M_{\mathrm{Bi}} \omega_{0}^{2} q_{0}=3.123 \times 10^{-6} \mathrm{erg} / \mathrm{cm}$.

The equation for atomic motion in a solid perturbed by the laser reads

$$
\frac{d^{2} q_{k}}{d t^{2}}+2 \gamma \frac{d q_{k}}{d t}+\omega_{0}^{2} q_{k}=\frac{F_{k}^{\text {las }}}{M} .
$$

Here, the phenomenological damping coefficient $\gamma$ is introduced; we discuss its physical meaning later in the paper. $F_{k}^{\text {las }}=F_{\mathrm{th}}+F^{(p)}+f^{\text {pond }}$ is the sum of the laser-exerted forces.

\section{Laser-induced atomic motion}

\section{Fast atomic displacement}

The relative influence of the elastic force and the laserexerted forces is different on short-time scales $t<\omega_{0}^{-1}$ and on long-time scales $t \gg \omega_{0}^{-1}$. The slow elastic force is not effective during the period much shorter than that of the atomic vibration. Correspondingly, the first term on the left-hand side of Eq. (9) dominates and it reduces to the simplest form of the Newton equation:

$$
\frac{d^{2} q_{k}}{d t^{2}} \cong \frac{F_{k}^{\text {las }}(t)}{M} .
$$

The solution of this equation is straightforward:

$$
q_{k}(t)=M^{-1} \int_{t^{\prime}}^{t} d t^{\prime} \int_{0}^{t^{\prime}} F_{k}^{\mathrm{las}}\left(t^{\prime \prime}\right) d t^{\prime \prime} \approx \frac{F_{\max }^{\mathrm{las}} t_{p}^{2}}{2 M} .
$$

Thus, on a short-time scale the laser-imposed forces produce a coherent displacement of atoms. It is instructive to compare the fast atomic displacement to the amplitude of cold phonons [Eq. (8)]. Taking the above parameters for the experiments with $\mathrm{Bi}$ one obtains that during $\sim 100$ fs bismuth atoms are displaced on the distance less than $10^{-10} \mathrm{~cm}$ which is approximately one tenth of the cold phonon's amplitude.

\section{Quasiharmonic vibrations under the action of thermal forces}

One can see that the maximum value of the sum of the laser-imposed forces equals to approximately one tenth of the elastic force driving cold phonons, $\omega_{0}^{2} q_{0} \gg F_{k}^{\text {las }} / M$. Thus on a long-time scale $t \gg \omega_{0}^{-1}$ the laser-imposed forces act as a small perturbation slightly affecting the cold atomic vibrations. Therefore, one can search for a solution of Eq. (9) in the form

$$
q_{k}(t)=q_{k 0}(t)+\Delta q_{k}(t),
$$

where $q_{0}$ is the unperturbed solution

$$
q_{k}=q_{k 0} \exp \left\{-i\left(\omega_{0}^{2}-\gamma^{2}\right)^{1 / 2} t\right\} .
$$

Now the equation for the perturbation can be expressed as follows:

$$
\frac{d^{2}\left(\Delta q_{k}\right)}{d t^{2}}+2 \gamma \frac{d\left(\Delta q_{k}\right)}{d t}+\omega_{0}^{2} \Delta q=\frac{F_{k}^{\text {las }}}{M} .
$$

We assume that the perturbation oscillates with the same frequency as the unperturbed vibration and therefore that the perturbation affects only the vibration amplitude. Thus, one obtains the change in the vibrational amplitude at $t \gg \omega_{0}^{-1}$ in the form

$$
\Delta q_{k}=\left\{F_{\mathrm{th}, k} / 2 \omega_{0} \gamma M\right\} \exp \left\{-\gamma t+i\left(\omega_{0}^{2}-\gamma^{2}\right)^{1 / 2} t-i \varphi\right\} .
$$

Note that only the thermal force is effective after the end of the pulse. The phase and some pre-exponential constant should be introduced in order to stitch the above solution to the initial atomic displacement.

\section{Nonlinear phenomena arising in the process of electron-to- lattice energy transfer and lattice heating}

a. Anharmonicity of atomic vibrations due to lattice heating: thermal expansion shift in equilibrium position. Above we considered damped harmonic oscillations of atoms where the electron and lattice temperature entered only into the thermal force. However, after the pulse the initially excited cold harmonic phonon modes are gradually transformed because the lattice temperature increases due to energy transfer from the electrons. Thus, after establishment of the lattice temperature, the phonon modes are distributed over the energy in accord with the Boltzmann distribution with temperature $T, \phi_{\mathrm{ph}}(q)=\exp \left\{-\Delta U_{e l} / T\right\}$. The phonon energy [harmonic perturbation in the interatomic potential (6)] satisfies the condition $\Delta U_{e l} \ll T$. Phonons do not interact in the harmonic approximation. Respectively, the equilibrium position of vibrating atoms does not depend on temperature, and the average displacement of an oscillating atom from the equilibrium position is zero. ${ }^{36}$ However, the mean square displacement (or the average phonon energy) is proportional to temperature:

$$
\left\langle q^{2}\right\rangle=\frac{\int_{-\infty}^{\infty} q^{2} \exp \left(-\Delta U_{e l} / T\right) d q}{\int_{-\infty}^{\infty} \exp \left(-\Delta U_{e l} / T\right) d q}=\frac{T}{M \omega_{\mathrm{ph}}^{2}} .
$$

This derivation qualitatively complies with a more rigorous calculation for the average amplitude of atomic vibrations 
around the lattice vertex at a temperature exceeding the Debye temperature $T_{D},{ }^{37,38} M \omega_{D}^{2}\left\langle q^{2}\right\rangle \approx T$. Here, $\omega_{D}=T_{D} / \hbar$ is the Debye frequency.

The phonon-phonon interactions become important when the growing lattice temperature approaches the equilibrium melting point. The atomic vibrations lose their harmonic character; the nonlinearity in the interaction potential should be taken into account. The perturbation in the interatomic potential with the third-order term included reads ${ }^{36}$

$$
\Delta U_{\mathrm{nl}}=C q^{2}-g q^{3}, \quad C \approx \varepsilon_{b} / 2 d^{2}, \quad g=\frac{1}{6}\left(\frac{\partial^{3} U}{\partial q^{3}}\right)_{0} \approx \frac{\varepsilon_{b}}{6 d^{3}} .
$$

The distribution function with the nonlinear term included is presented as the following: ${ }^{37}$

$$
\phi_{\mathrm{nl}}=\exp \left\{-\left(C q^{2}-g q^{3}\right) / T\right\} \approx \exp \left\{-C q^{2} / T\right\}\left(1+g q^{3} / T\right) .
$$

Now the average displacement from the cold equilibrium position increases as the lattice temperature goes up:

$$
\left\langle q_{\mathrm{nl}}\right\rangle=\frac{3 g T}{4 C^{2}} \approx \frac{T}{2 \varepsilon_{b}} d .
$$

The atomic motion loses its oscillatory character and becomes chaotic (randomized) when the change in average atomic position by Eq. (19) constitutes a significant part of the oscillation amplitude. The displacement of equilibrium atomic position due to nonlinear interaction of phonons calculated by Eq. (19) at a temperature equal to that of the equilibrium melting point coincides with the average displacement following from the Lindemann criterion of melting with an accuracy of $10 \% .^{38,39}$ This seems to be an amazing coincidence from the first sight. In fact, it is the evidence for the failure of the harmonic approximation for description of atomic motion during the phase transition stage.

The nonlinear processes of multiphonon interaction at temperatures close to the equilibrium melting point gradually results first in the mode softening (that is the decrease of the oscillation frequency), and then in the lattice instability later in time when the square of the frequency may turn negative.

Now we define the time scale when the nonlinear interaction becomes dominant.

b. Three-phonon interaction: phonon decay time (lifetime). The probability of multiphonon processes in unit time defines the characteristic phonon decay time. The probability of decay of an optical phonon (spatial dispersion neglected) into two acoustic phonons per unit time can be calculated with the help of quantum perturbation theory as third-order term. ${ }^{31}$ We present here a simplified version of a similar derivation estimating the perturbation Hamiltonian, $H^{\prime}$, as the third term in the series of potential expansion in powers of atomic displacement from Eq. (16). Then the scaling for the probability for the phonon decay is the following:

$$
w \propto \frac{\left|H^{\prime}\right|^{2}}{\hbar T},
$$

where we take the average phonon energy as $M \omega_{D}^{2}\left\langle q^{2}\right\rangle$ $\approx \hbar \omega_{\mathrm{ph}} \approx T$. Respectively, the third-order term in the perturbation potential (perturbation Hamiltonian) is expressed as follows:

$$
H^{\prime} \approx \frac{1}{6}\left(\frac{\partial^{3} U}{\partial q^{3}}\right)_{0}\left\langle q^{2}\right\rangle^{3 / 2} \approx \frac{\varepsilon_{b}}{6 d^{3}}\left(\frac{T}{M \omega_{D}^{2}}\right)^{3 / 2} .
$$

Now the probability of phonon decay, or the optical phonon decay rate, can be easily expressed as function of basic properties of a solid. For temperatures in excess of the Debye temperature, $k T \gg \hbar \omega_{D}$, the single phonon decay rate reads

$$
w \approx C \frac{\hbar}{M d^{2}}\left(\frac{k T}{\hbar \omega_{D}}\right)^{2},
$$

where $C$ is a proportionality parameter in the scaling law. For Bi $\left(M_{\mathrm{Bi}}=3.47 \times 10^{-22} \mathrm{~g} ; d=3.3 \times 10^{-8} \mathrm{~cm}\right)$ at $k T=\hbar \omega_{D}$ $\left(T_{D}=119 \mathrm{~K}\right)$ this decay rate equals to $w \approx \hbar / M d^{2}=2.79$ $\times 10^{9} \mathrm{~s}^{-1}$. Respectively, at the maximum lattice temperature of $1273 \mathrm{~K}$ (at $6.7 \mathrm{~mJ} / \mathrm{cm}^{2}$ ) this rate is $3.2 \times 10^{11} \mathrm{~s}^{-1}$, which is in qualitative agreement with the experimental observations. Nonlinear phonon-phonon interactions also result in the dependence of the phonon frequency on the lattice temperature.

c. Redshift in the phonon frequency and increase in the interatomic separation due to laser excitation. The binding energy in the laser-excited solid is reduced compared to that in a cold state due to the excitation of electrons and the lattice heating. One can describe the property of excited solid using a simplified form for the empirical chemical pseudopotential: ${ }^{40,41}$

$$
V(r)=V_{R} e^{-\theta r}-V_{A} e^{-\lambda r} .
$$

Here $V_{R}, V_{A}, \theta$, and $\lambda$ (or $s=\theta / \lambda$ and $\lambda$ ) are, respectively, the repulsive and attractive potentials along with their gradients. The attractive part of the interatomic potential decreases with temperature increase in comparison to the repulsive part, thus effectively increasing the repulsion. It follows from Eq. (23) that in the heated solid the interatomic distance (where the potential is a minimum) can be expressed via the binding energy as the following:

$$
d=\frac{1}{\lambda} \ln \left(\frac{(s-1) V_{A}}{s \varepsilon_{b}}\right) \approx d_{0}+\frac{T}{\lambda \varepsilon_{b 0}} .
$$

Thus in the excited state at $\varepsilon_{b}-T_{e}$ (assuming $\varepsilon_{b} \gg T_{e}$ ) the interatomic distance increases and this increase depends on the gradient of the attractive part of the potential that is most strongly affected:

$$
d q \approx \frac{1}{\lambda} \frac{T_{e}}{\varepsilon_{b, 0}} .
$$

One can see that the above relation qualitatively complies with Eq. (18) and that it is the result of thermal expansion. However, in Eq. (25) the asymmetry in the interatomic po- 
tential is taken into account. Similarly, the second derivative of the potential allows recovery of the excited phonon frequency [compare to Eq. (6)]:

$$
\omega_{\mathrm{ph}}^{2}=\frac{1}{M}\left(\frac{\partial^{2} V}{\partial r^{2}}\right)_{r=d}=\frac{\lambda^{2} s\left|\varepsilon_{b}\right|}{M} .
$$

Thus the square of the phonon frequency in the excited solid linearly decreases with temperature growth in the first approximation, where the change in the interatomic distance with the temperature is ignored:

$$
\omega_{\mathrm{ph}}^{2} \approx \omega_{0}^{2}\left(1-\frac{T_{e}}{\left|\varepsilon_{b 0}\right|}\right) .
$$

Therefore, the above estimate implicitly assumes that electrons are excited and the lattice is cold. For example, in $\mathrm{Bi}$ excited to the maximum electron temperature of $3480 \mathrm{~K}$ (which is $0.3 \mathrm{eV}$, while the binding energy $2.16 \mathrm{eV}$ ) the phonon frequency decrease predicted by Eq. (27) is $\sim 7 \%$. Thus for the $A_{1 g}$ mode the frequency of $3 \mathrm{THz}$ in accord to Eq. (27) should decrease to $2.78 \mathrm{THz}$. The experimental value is $2.82 \mathrm{THz}$ at $4 \mathrm{~mJ} / \mathrm{cm}^{2}$ (see Fig. 5). One can see that when the temperature approaches the equilibrium melting point, which is the onset of transition to the intermediate potential minimum corresponding to liquid state, the second derivative of the potential passes through zero. This is a manifestation of the onset of vibrational instability when the square of phonon frequency temporarily may turn negative.

\section{Transient optical properties in the laser-excited solid}

\section{Dielectric function}

Our goal is to trace the atomic motion and the subsequent phase changes through the time-dependent dielectric properties of laser-affected material. We assumed in Eq. (2) that the dielectric tensor modified by the laser effect consists of two terms, the Drude-type term, $\varepsilon_{D}$, and the polarization term, $\varepsilon_{j k}^{(p)}: \varepsilon_{j k}=\varepsilon_{D} \cdot \delta_{j k}+\varepsilon_{j k}^{(p)}$. The polarization part is the real number. Thus, the total dielectric function can be presented as a sum:

$$
\begin{gathered}
\varepsilon_{j k}=\varepsilon_{D} \cdot \delta_{j k}+\varepsilon_{j k}^{(p)} \equiv \varepsilon_{r}+i \varepsilon_{i}, \\
\varepsilon_{r}=\varepsilon_{j k}^{(p)}+\varepsilon_{r, D}, \quad \varepsilon_{i} \equiv \varepsilon_{i, D} .
\end{gathered}
$$

The Drude term has its conventional form:

$$
\varepsilon_{D}=1-\frac{\omega_{p}^{2}}{\omega^{2}+\nu_{e-\mathrm{ph}}^{2}}+i \frac{\omega_{p}^{2}}{\omega^{2}+\nu_{e-\mathrm{ph}}^{2}} \frac{\nu_{e-\mathrm{ph}}}{\omega} \equiv \varepsilon_{r, D}+i \varepsilon_{i, D} .
$$

This function depends on the number density of the conductivity electrons, $n_{e}$, and on the electron effective mass through plasma frequency, $\omega_{p}^{2}=\frac{4 \pi e^{2} n_{e}(t)}{m_{e}^{*}}$. It is unknown to the best of our knowledge how the electron effective mass changes during heating and melting of a solid. For example, it is established that in liquid bismuth, the effective mass is equal to that of a free electron's; ${ }^{42}$ we may assume that in the solid phase at room temperature the effective mass is also equal to the free electron mass. Therefore we ignore the electron effective mass change in the following analysis. The dependence of the electron-phonon energy exchange on the phonon amplitude and frequency and on the lattice temperature enters through the electron-phonon momentum exchange rate in the explicit form with the use of Eqs. (8) and (18):

$$
\nu_{e-\mathrm{ph}} \approx \varepsilon_{\mathrm{ph}} / \hbar=M \omega_{D}^{2} q^{2} / 2 \hbar=\omega_{D} q^{2} / 2 q_{0}^{2} .
$$

The small perturbation in the electron-phonon rate due to laser excitation is expressed through the change in the phonon amplitude as follows:

$$
\Delta \nu_{e-\mathrm{ph}} \approx \nu_{e-\mathrm{ph}}^{(0)} \frac{2 \Delta q(t)}{q_{0}} .
$$

The polarizability part from Eq. (4) is expressed directly through the laser-induced atomic displacement:

$$
\varepsilon_{i k}^{(p)}=4 \pi\left(\frac{\partial \chi_{i k}}{\partial x_{l}}\right)_{0} \Delta q_{l}(t) .
$$

The atomic displacement $\Delta q(t)$ is expressed by formula (15).

The real and imaginary parts of the slightly perturbed dielectric function are as follows:

$$
\begin{gathered}
\Delta \varepsilon_{r}=\Delta \varepsilon_{j k}^{(p)}+\Delta \varepsilon_{r, D}=\Delta \varepsilon_{j k}^{(p)}+\left(\frac{\partial \varepsilon_{D, r}}{\partial \ln n_{e}}\right) \frac{\Delta n_{e}}{n_{e}} \\
+\left(\frac{\partial \varepsilon_{D, r}}{\partial \ln \nu_{e-\mathrm{ph}}}\right) \frac{\Delta \nu_{e-\mathrm{ph}}}{\nu_{0, e-\mathrm{ph}}} \\
\Delta \varepsilon_{i} \equiv \Delta \varepsilon_{i, D}=\left(\frac{\partial \varepsilon_{i, D}}{\partial \ln n_{e}}\right) \frac{\Delta n_{e}}{n_{e}}+\left(\frac{\partial \varepsilon_{i, D}}{\partial \ln \nu_{e-\mathrm{ph}}}\right) \frac{\Delta \nu_{e-\mathrm{ph}}}{\nu_{0, e-\mathrm{ph}}} .
\end{gathered}
$$

Subscript " 0 " denotes that a derivative is taken from the unperturbed function. Derivatives are presented in the Appendix. Thus, the dielectric function in a swiftly excited metal-like solid is changing due to the increase in the number density of conductivity electrons and the variation in the electron-phonon momentum exchange rate. The transient changes following the excitation are expressed as explicit functions of the electron temperature and atomic vibrations.

For example, in $\mathrm{Bi}$ the real part of the dielectric function in equilibrium liquid is slightly higher than that in a solid while the imaginary part increases more than two times. The number density of electrons and the electron-phonon rate in liquid are almost three times higher of those in a solid (see Ref. 42 and Table I below). On the other hand, the logarithmic derivatives of the real and imaginary parts of the dielectric function on electron number density and derivatives of real parts on the electron-phonon rate are of the same sign in both states (see Table II below). However, the logarithmic derivative of the imaginary part on the electron-phonon rate in a solid is slightly positive (0.86) while in a liquid it is strongly negative $(-11.95)$. Thus, the sign of this derivative changes somewhere during the solid-liquid transition time. One can make two conclusions from this analysis. First, one 
TABLE I. Solid and liquid bismuth optical properties at $800 \mathrm{~nm}\left(\omega=2.356 \times 10^{15} \mathrm{~s}^{-1}\right.$, photon energy 1.55 $\mathrm{eV})$.

\begin{tabular}{lcccccccc}
\hline \hline & $\varepsilon_{\text {real }}$ & $\varepsilon_{\text {im }}$ & $\varepsilon$ & $n_{e}$ & $m_{e}$ & $v_{\text {eff }} / \omega$ & $R_{0}$ & $\left(\omega_{p} / \omega\right)^{2}$ \\
\hline Solid $^{\mathrm{a}}$ & -16.25 & 15.4 & 22.39 & $5.34 \times 10^{22}$ & Free & 0.893 & 0.74 & 31.0 \\
Liquid $^{\mathrm{b}}$ & -11.0 & 28.9 & 30.92 & $14.1 \times 10^{22}$ & Free & 2.408 & 0.67 & 81.58 \\
\hline \hline
\end{tabular}

References 22-24

${ }^{b}$ Reference 42 .

cannot present the transient dielectric function as an expansion into series during the solid-liquid transition because linear approximation does not work in this case. Second, the transient phase state may have peculiar optical properties due to strong changes in the electron-phonon collision rate directly related to the laser-excited atomic motion.

\section{Time-dependent reflectivity}

Now it is instructive to present the directly measured time-dependent reflectivity of a laser-excited solid through the solid internal properties. First, we express the Fresnel reflection coefficient, $R=\left|\varepsilon^{1 / 2}-1 / \varepsilon^{1 / 2}+1\right|^{2}$, through the real and imaginary parts of the dielectric function, $\varepsilon=\varepsilon^{(r)}+i \varepsilon^{(i)}$, as follows:

$$
R=\frac{|\varepsilon|+1-2^{1 / 2}\left(|\varepsilon|+\varepsilon_{r}\right)^{1 / 2}}{|\varepsilon|+1+2^{1 / 2}\left(|\varepsilon|+\varepsilon_{r}\right)^{1 / 2}} .
$$

Here $|\varepsilon|=\left(\varepsilon_{r}^{2}+\varepsilon_{i}^{2}\right)^{1 / 2}$. Small variation in reflectivity then reads

$$
\Delta R=\left(\frac{\partial R}{\partial \varepsilon_{r}}\right)_{0}\left(\Delta \varepsilon_{r, D}+\Delta \varepsilon_{j k}^{(p)}\right)+\left(\frac{\partial R}{\partial \varepsilon_{i}}\right)_{0} \Delta \varepsilon_{i, D} .
$$

Substituting variations in the dielectric function from Eq. (33) one can present the small first-order reflectivity variation expressed through the changes in polarization, in the number of the conductivity electrons and in the electronphonon rate with the coefficients combined through the unperturbed solid parameters:

$$
\Delta R=\left(\frac{\partial R}{\partial \varepsilon_{r}}\right)_{0} \Delta \varepsilon_{j k}^{(p)}+C_{n_{e}} \frac{\Delta n_{e}}{n_{e, 0}}+C_{\nu_{e-\mathrm{ph}}} \frac{\Delta \nu_{e-\mathrm{ph}}}{\nu_{e-\mathrm{ph}, 0}} .
$$

Here the coefficients (see the Appendix) are combined from the derivatives taken from the unperturbed functions:

$$
\begin{gathered}
C_{n_{e}}=\left(\frac{\partial R}{\partial \varepsilon_{r}}\right)_{0}\left(\frac{\partial \varepsilon_{r}}{\partial \ln n_{e}}\right)_{0}+\left(\frac{\partial R}{\partial \varepsilon_{i}}\right)_{0}\left(\frac{\partial \varepsilon_{i}}{\partial \ln n_{e}}\right)_{0}, \\
C_{\nu_{e-\mathrm{ph}}}=\left(\frac{\partial R}{\partial \varepsilon_{r}}\right)_{0}\left(\frac{\partial \varepsilon_{r}}{\partial \ln \nu_{e-\mathrm{ph}}}\right)_{0}+\left(\frac{\partial R}{\partial \varepsilon_{i}}\right)_{0}\left(\frac{\partial \varepsilon_{i}}{\partial \ln \nu_{e-\mathrm{ph}}}\right)_{0} .
\end{gathered}
$$

For Bi (see III below and the Appendix) both coefficients in expansion (36) have the same signs $\left(C_{n_{e}}\right.$ is positive and $C_{v_{e-\mathrm{ph}}}$ is negative) and they are of the same order of magnitude in both solid and liquid states. It is also known from the experiments $^{2-8,11,12,14,24}$ that the amplitude of the reflectivity changes in the observed oscillations is on the order of
$10^{-3}-10^{-4}$. Therefore, it is reasonable to describe the timedependent reflectivity oscillations by expansion (36) where each term has a clear physical meaning. Indeed, the first term describes the reflectivity decrease due to polarization changes in the dielectric function during the pulse, which is positive, while the reflectivity derivative is negative (for $\mathrm{Bi}$ ). The second term describes the increase in the reflectivity during the laser pulse that is the consequence of the increase in the number of conductivity electrons. After the end of the pulse this contribution gradually decreases (remaining positive) due to the recombination of excited carriers. The third contribution into the reflectivity changes is the result of the effect of atomic vibrations on the optical properties of excited solid. This contribution is of the same physical nature as the first term and it is negative. All variations in optical properties are explicit functions of electron and lattice temperature, which are functions of laser excitation and parameters of the solid.

\section{COMPARISON TO THE EXPERIMENTS}

The reflectivity of a probe beam was recorded with time resolution of $100 \mathrm{fs}$ and less in many experiments, $1,9,43$ where the absorbed energy density has been several times larger the equilibrium enthalpy of melting. Under these conditions a solid transferred into a transient state.

What kind of information about the transient phase state of laser-excited solid can be extracted from the timedependent reflectivity oscillations? First of all, the reflectivity of the transient state has to be compared to the reflectivity of the solid and liquid states. The reflectivity increases first over the level for a cold solid after the end of the pulse to its maximum value during the time comparable to the cold phonon period. Then, the reflectivity starts to oscillate with the cold phonon frequency and the amplitude gradually decreasing with time. In several picoseconds time, during a period comparable to the electron-phonon energy transfer time the reflectivity decreases below the initial solid level indicating the onset of the phase transition to a liquid state but never reaches the liquid value. Another distinctive feature of the transient reflectivity is a time period in which reflectivity oscillations cease to exist. This time is associated with lifetime of atomic harmonic vibrations, or phonons, it can be directly measured from the time-dependent reflectivity as an inverse of the damping coefficient. As the above analysis suggests, this time is inversely proportional to the probability per unit time for the optical phonon to decay into two acoustic phonons. Thus this time signifies the moment when harmonic atomic oscillations are completely converted into a 
TABLE II. Numerical values for dielectric constant derivatives for solid and for liquid Bi at $800 \mathrm{~nm}$.

\begin{tabular}{lcccccc}
\hline \hline & $\partial \varepsilon_{r} / \partial \ln n_{e}$ & $\partial \varepsilon_{r} / \partial \ln \nu_{e-\mathrm{ph}}$ & $\partial \varepsilon_{i} / \partial \ln n_{e}$ & $\partial \varepsilon_{i} / \partial \ln \nu_{e-\mathrm{ph}}$ & $d R / d \varepsilon_{r}$ & $d R / d \varepsilon_{i}$ \\
\hline Solid Bi & -17.25 & 0.032 & 15.4 & 0.87 & $-1.2 \times 10^{-2}$ & $-6 \times 10^{-3}$ \\
Liquid Bi & -12 & 0.012 & 28.9 & -11.94 & $-7.5 \times 10^{-3}$ & $1.58 \times 10^{-3}$ \\
\hline \hline
\end{tabular}

nonlinear chaotic motion. For the considered case where the temperature is higher than the Debye temperature $k T \gg k T_{D}$ the single phonon decay rate reads

$$
w_{\text {decay }} \approx C \frac{\hbar}{M d^{2}}\left(\frac{k T}{\hbar \omega_{0}}\right)^{2},
$$

where $C$ is a proportionality parameter in the scaling law. For $\mathrm{Bi} \quad\left(M_{\mathrm{Bi}}=3.47 \times 10^{-22} \mathrm{~g} ; \quad d=4 \times 10^{-8} \mathrm{~cm} ; \quad \omega_{0}=1.77\right.$ $\times 10^{12} \mathrm{~s}^{-1}$; unperturbed phonon energy $1.17 \times 10^{-3} \mathrm{eV}$ ) at low temperature, below the Debye temperature, this decay rate equals to $1.89 \times 10^{9} \mathrm{~s}^{-1}$. Examination of the experimental data in Fig. 3 shows that the decay time follows a temperature power law of 1.4, which is lower than the temperature-squared law for a single phonon decay. This suggests that the two-phonon decay process was mixed with some other involved dissipation processes, such as multiphonon interaction. In other words, the moment when the reflectivity oscillation amplitude ceases to exist can be considered as the onset of the vibration instability, which in turn instigates the beginning of the disordering of a solid crystal and the beginning of the transition. Reflectivity oscillations in $\mathrm{Bi}$ were measured at different pump fluences, i.e., at different electron and lattice temperatures. These oscillations were also observed for different initial temperatures at the same excitation fluence.

It was observed that the phonon frequency decreases (redshifts) compared to the cold material with the increase in the pump fluence, i.e., with the growth of maximum electron and lattice temperature in the excited solid at the constant initial temperature of a sample. The redshift of phonon frequency was also observed as a function of the initial lattice temperature while the excitation fluence remained the same. However the magnitude of the shift in these two cases is different.

In a solid heated in the equilibrium conditions (electron and lattice temperatures are the same) the binding energy decreases along with the increase in the interatomic distance due to thermal expansion (with the shift in the equilibrium positions of vibrating atoms). The phonon frequency scales with the binding energy and the interatomic distance as $\omega_{\mathrm{ph}}^{2}$ $\approx \varepsilon_{b} / M d^{2}$. Therefore, changes in the interatomic distance, $d \approx d_{0}+T /\left(\lambda \varepsilon_{b 0}\right)$, and in the binding energy, $\varepsilon_{b} \approx \varepsilon_{b 0}-T$, both contribute into the frequency change as

$$
\omega_{\mathrm{ph}}^{2} \approx \omega_{\mathrm{ph}, 0}^{2}\left(1-\frac{T}{\varepsilon_{b, 0}}\right)\left(1+\frac{T}{\lambda d_{0} \varepsilon_{b, 0}}\right)^{-2} .
$$

Keeping only the first-order terms in expansion of the above squared frequency in series of a small parameter, $T / \varepsilon_{b 0}$, one obtains the redshift of the phonon frequency as a function of temperature in the form

$$
\omega_{\mathrm{ph}}^{2} \approx \omega_{\mathrm{ph}, 0}^{2}\left\{1-\left(1-\frac{2}{\lambda d_{0}}\right) \frac{T}{\varepsilon_{b, 0}}\right\} .
$$

With swift laser heating the frequency redshift occurs mainly due to the rise in the electron temperature because the coherent displacement of atoms during the short pulse does not change the interatomic distance in a cold lattice. Therefore the frequency change by the action of the ultrashort pulse is expressed as

$$
\omega_{\mathrm{ph}}^{2} \approx \omega_{\mathrm{ph}, 0}^{2}\left\{1-\left(T_{e}+T_{0}\right) / \varepsilon_{b, 0}\right\} .
$$

Comparing Eqs. (39) and (40) one can see that at the same temperature the frequency shift in equilibrium might be several times larger than that during the subpicosecond excitation. However, this linear approximation is only the indication of a trend. When the temperature approaches the melting point, nonlinear effects may become dominant and the above expansions will be an underestimate.

The dependence of the frequency of reflectivity oscillations in the bismuth sample at room temperature $\left(T_{0}\right.$ $=290 \mathrm{~K}, 0.025 \mathrm{eV}$ ) as a function of the exciting laser fluence, which is presented in Fig. 5, is well described by Eq. (40) with $\omega_{\mathrm{ph}, 0}=3.04 \mathrm{THz}$ up to the fluence of $\sim 13 \mathrm{~mJ} / \mathrm{cm}^{2}$ when maximum electron temperature reaches $0.573 \mathrm{eV}$, and maximum lattice temperature is 4.5 times higher than equilibrium melting point. It is amazing that a simple linear approximation works so well even outside the area of its applicability. Similarly, the oscillation frequency dependence on the initial temperature in a range from 50 to $510 \mathrm{~K}$ was measured at a fixed fluence of $6.9 \mathrm{~mJ} / \mathrm{cm}^{2}$ (maximum electron temperature $0.363 \mathrm{eV}$ ). The simple formulae of Eqs. (39) and (40) explain well the experimental data in the range of applicability of the linear approximation. Experiments at the laser fluence level leading to strong overheating (fluence $>10 \mathrm{~mJ} / \mathrm{cm}^{2}, T_{L}>3.5 T_{\text {melt }}$ ) show much stronger influence of nonlinear effects than those taken into account by the first-order approximation by Eqs. (39) and (40).

\section{CONCLUSIONS}

Summing up one can conclude that reflectivity oscillations measured with high accuracy allow subtle atomic motion, induced by the action of the laser pulse with duration shorter than all relaxation times, to be uncovered. First, the coherent atomic displacement is produced by the polarization force and electron pressure force during the laser pulse and this results in the initial negative drop in the reflectivity. The frequency of the following reflectivity oscillations is chirped, redshifting from the initial value due to electron heating. The amplitude decreases gradually as the electrons transfer their energy to the lattice. Heating of the lattice and thermal ex- 
pansion transformed the initially harmonic vibrations of the atoms into strongly nonlinear chaotic motion that is characteristic of the onset of solid-to-liquid phase transformation. This process is identified by the measurement and interpretation of the damping rate of the reflectivity oscillations. The observed dependence of the damping rate on the temperature is close to the dependence of the rate of optical phonon decay into two acoustic phonons, and this confirms the interpretation of the inverse damping rate as the lifetime of the phonons. The reflectivity oscillations are followed by a decrease below the reflectivity of the initial solid state toward the liquid state. However, the level of this decrease before recovering to the initial solid reflectivity is relatively small, $\Delta R / R_{0} \leq 5 \times 10^{-3}$ at most, while the difference between the reflectivity of solid and liquid phases is much larger: $\left(R_{\text {solid }}\right.$ $\left.-R_{\text {liq }}\right) / R_{\text {solid }}=0.1$. It seems from the naïve point of view that melting should have occurred inevitably at a lattice temperature in excess of melting point. However it appears to be wrong in the case of ultrafast heating. Indeed, it has been demonstrated that ice, ${ }^{44}$ gallium, ${ }^{45}$ and aluminum ${ }^{43,46}$ overheated to $T_{\max } / T_{\text {melt }}=1.073,2.67,9.4$ do not melt for times of $200 \mathrm{ps}, 20 \mathrm{ps}$ and $3.5 \mathrm{ps}$, respectively, where this time is much longer than electron-lattice equilibration time. The ratio of maximum temperature to the melting point in the experiments presented in this paper and in Ref. 24 is $1273 \mathrm{~K} / 545 \mathrm{~K}=2.33$. Thus, the results of this paper are in agreement with Refs. 43-46. There were no indications that the phase transition was completed during several tens of picoseconds after the laser excitation. A deeper insight into internal properties of a solid in this transition state is needed in order to describe it in more detail (the distribution functions, order parameter, etc.).

\section{ACKNOWLEDGMENTS}

The support of Programme International de Cooperation
TABLE III. Coefficients in the reflectivity variations.

\begin{tabular}{lcc}
\hline \hline & $C_{n_{e}}$ & $C_{v_{e-p h}}$ \\
\hline Solid $^{\mathrm{a}} \mathrm{Bi}$ & +0.1146 & $-5.6 \times 10^{-3}$ \\
Liquid $^{\mathrm{b}} \mathrm{Bi}$ & +0.1357 & $-1.9 \times 10^{-2}$ \\
\hline
\end{tabular}

${ }^{\mathrm{a}}$ References 22-24.

${ }^{\mathrm{b}}$ Reference 42 .

Scientifique (PICS, France) is gratefully acknowledged. T.G. and D.B. acknowledge support of the European Community Marie Curie research training network FLASH (Grant No. MRTN-CT-2003-503641).

\section{APPENDIX}

The derivatives of the Drude dielectric function are the following:

$$
\begin{gathered}
\frac{\partial \varepsilon_{r}}{\partial \ln n_{e}}=\varepsilon_{r}-1, \quad \frac{\partial \varepsilon_{r}}{\partial \ln \nu_{e-\mathrm{ph}}}=2 \varepsilon_{i}^{2} \frac{\omega^{2}}{\omega_{p}^{2}}, \\
\frac{\partial \varepsilon_{i}}{\partial \ln n_{e}}=\varepsilon_{i}, \quad \frac{\partial \varepsilon_{i}}{\partial \ln \nu_{e-\mathrm{ph}}}=\varepsilon_{i} \cdot \frac{1-(\nu / \omega)^{2}}{1+(\nu / \omega)^{2}} .
\end{gathered}
$$

Below are the reflectivity derivatives from the Fresnel formula expressed through the dielectric function:

$$
\begin{aligned}
& \frac{d R}{d \varepsilon_{r}}=\frac{\sqrt{2}\left[\varepsilon_{r}^{2}-\varepsilon_{i}^{2}-|\varepsilon|+\varepsilon_{r}(|\varepsilon|-1)\right]}{|\varepsilon| \sqrt{\left(|\varepsilon|+\varepsilon_{r}\right)}\left[|\varepsilon|+1+\sqrt{2\left(|\varepsilon|+\varepsilon_{r}\right)}\right]^{2}}, \\
& \frac{d R}{d \varepsilon_{i}}=\frac{\varepsilon_{i}}{|\varepsilon|} \frac{\sqrt{2}\left(|\varepsilon|+2 \varepsilon_{r}-1\right)}{\sqrt{\left(|\varepsilon|+\varepsilon_{r}\right)}\left[|\varepsilon|+1+\sqrt{2\left(|\varepsilon|+\varepsilon_{r}\right)}\right]^{2}} .
\end{aligned}
$$

Here $|\varepsilon|=\left(\varepsilon_{r}^{2}+\varepsilon_{i}^{2}\right)^{1 / 2}$. Both derivatives are negative for solid $\mathrm{Bi}$ at $800 \mathrm{~nm}$ (Tables II and III). *gam111@rsphysse.anu.edu.au

${ }^{1}$ A. Rousse, C. Rischel, S. Fourmaux, I. Uschmann, S. Sebban, G. Grillon, Ph. Balcou, E. Foerster, J. P. Geindre, P. Audebert, J. C. Gauthier, and D. Hulin, Nature (London) 410, 65 (2001).

${ }^{2}$ H. J. Zeiger, J. Vidal, T. K. Cheng, E. P. Ippen, G. Dresselhaus, and M. S. Dresselhaus, Phys. Rev. B 45, 768 (1992).

${ }^{3}$ T. K. Cheng, J. Vidal, H. J. Zeiger, G. Dresselhaus, M. S. Dresselhaus, and E. P. Ippen, Appl. Phys. Lett. 59, 1923 (1991).

${ }^{4}$ R. Merlin, Solid State Commun. 102, 207 (1997).

${ }^{5}$ G. A. Garrett, T. F. Albrecht, J. F. Whitaker, and R. Merlin, Phys. Rev. Lett. 77, 3661 (1996).

${ }^{6}$ M. Hase, K. Mizoguchi, H. Harima, S. Nakashima, M. Tani, K. Sakai, and M. Hangyo, Appl. Phys. Lett. 69, 2474 (1996).

${ }^{7}$ É. D. Murray, D. M. Fritz, J. K. Wahlstrand, S. Fahy, and D. A. Reis, Phys. Rev. B 72, 060301(R) (2005).

${ }^{8}$ K. Ishioka, M. Kitajima, and O. V. Misochko, J. Appl. Phys. 100, 093501 (2006).

${ }^{9}$ K. Sokolowski-Tinten, C. Blome, J. Blums, A. Cavalleri, C. Dietrich, A. Tarasevitch, I. Uschmann, E. Foerster, M. Kammler,
M. Horn-von-Hoegen, and D. von der Linde, Nature (London) 422, 287 (2003).

${ }^{10}$ D. M. Fritz, D. A. Reis, B. Adams, R. A. Akre, J. Arthur, C. Blome, P. H. bucksbaum, A. L. Cavalieri, S. Engemann, S. Fahy, R. W. Falcone, P. H. Fuoss, K. J. Gaffney, M. J. George, J. Hajdu, M. P. Hertlein, P. B. Hillyard, M. Horn-von Hoegen, M. Kammler, J. Kaspar, R. Kienberger, P. Krejcik, S. H. Lee, A. M. Lindenberg, B. McFarland, D. Meyer, T. Montagne, É. D. Murray, A. J. Nelson, M. Nicoul, R. Pahl, D. von der Linde, and J. B. Hastings, Science 315, 633 (2007).

${ }^{11}$ O. V. Misochko, M. Hase, K. Ishioka, and M. Kitajima, Phys. Rev. Lett. 92, 197401 (2004).

${ }^{12}$ O. V. Misochko, R. Lu, M. Hase, and M. Kitajima, J. Exp. Theor. Phys. 104, 245 (2007).

${ }^{13}$ S. L. Johnson, P. Beaud, C. J. Milne, F. S. Krasniqi, E. S. Zijlstra, M. E. Garcia, M. Kaiser, D. Grolimund, R. Abela, and G. Ingold, Phys. Rev. Lett. 100, 155501 (2008).

${ }^{14}$ A. Q. Wu and X. Xu, Appl. Phys. Lett. 90, 251111 (2007).

${ }^{15}$ A. Cavalleri, Cs. Tóth, C. W. Siders, J. A. Squier, F. Ráksi, P. 
Forget, and J. C. Kieffer, Phys. Rev. Lett. 87, 237401 (2001).

${ }^{16}$ E. Collet, M.-H. Lemee-Cailleau, M. B.-L. Cointe, H. Cailleau, M. Wulff, T. Luty, S.-Y. Koshihara, M. Meyer, L. Toupet, P. Rabiler, and S. Techert, Science 300, 612 (2003).

${ }^{17}$ T. Dumitrica, M. E. Garcia, H. O. Jeschke, and B. I. Yakobson, Phys. Rev. Lett. 92, 117401 (2004).

${ }^{18}$ M. Chollet, L. Cuerin, N. Uchida, S. Fukaya, H. Shimoda, T. Ishikawa, K. Matsuda, T. Hasegawa, A. Ota, H. Yamochi, G. Saito, R. Tazaki, S.-I. Adachi, and S.-Y. Koshihara, Science 307, 86 (2005).

${ }^{19}$ A. J. Kent, R. N. Kini, N. M. Stanton, M. Henini, B. A. Glavin, V. A. Kochelap, and T. L. Linnik, Phys. Rev. Lett. 96, 215504 (2006).

${ }^{20}$ K. Ishioka, M. Hase, M. Kitajima, and H. Petek, Appl. Phys. Lett. 89, 231916 (2006).

${ }^{21}$ G. C. Cho, W. Kütt, and H. Kurz, Phys. Rev. Lett. 65, 764 (1990).

${ }^{22}$ M. Hase, K. Ishioka, J. Demsar, K. Ushida, and M. Kitajima, Phys. Rev. B 71, 184301 (2005).

${ }^{23}$ W. Albrecht, Th. Kruse, and H. Kurz, Phys. Rev. Lett. 69, 1451 (1992).

${ }^{24}$ D. Boschetto, E. G. Gamaly, A. V. Rode, B. Luther-Davies, D. Glijer, T. Garl, O. Albert, A. Rousse, and J. Etchepare, Phys. Rev. Lett. 100, 027404 (2008).

${ }^{25}$ M. F. DeCamp, D. A. Reis, P. H. Bucksbaum, and R. Merlin, Phys. Rev. B 64, 092301 (2001).

${ }^{26}$ P. B. Allen, Phys. Rev. Lett. 59, 1460 (1987).

${ }^{27}$ T. Garl, Ph.D. thesis, Ecole Polytechnique, Palaiseau, 2008.

${ }^{28}$ American Institute of Physics Handbook, 3rd ed., edited by D. E. Gray (McGraw-Hill, New York, 1972).

${ }^{29}$ Numerical data and Functional Relationships in Science and Technology, Landolt-Börnstein, New Series, Group III, Vol. 17, edited by O. Madelung, M. Schulz, and H. Weiss (SpringerVerlag, Berlin, 1983).

${ }^{30}$ E. M. Lifshitz and L. P. Pitaevskii, Physical Kinetics (Pergamon, Oxford, 1981).

${ }^{31}$ Yu. A. Il'inskii and L. V. Keldysh, Electromagnetic Response of Material Media (Plenum, New York, 1994).

${ }^{32}$ M. I. Kaganov, I. M. Lifshitz, and L. V. Tanatarov, Zh. Eksp. Teor. Fiz. 31, 232 (1956) [Sov. Phys. JETP 4, 173 (1957)].

${ }^{33}$ E. G. Gamaly, A. V. Rode, B. Luther-Davies, and V. T. Tikhonchuk, Phys. Plasmas 9, 949 (2002).

${ }^{34}$ L. D. Landau, E. M. Lifshitz, and L. P. Pitaevskii, Electrodynamics of Continuous Media (Pergamon, Oxford, 1984).

${ }^{35}$ Y. R. Shen and N. Bloembergen, Phys. Rev. 137, A1787 (1965).

${ }^{36}$ C. Kittel, Introduction to Solid State Physics (Wiley, New York, 1996).

${ }^{37}$ J. M. Ziman, Electrons and Phonons (Oxford University Press, London, 1960).

${ }^{38}$ David Pines, Elementary Excitations in Solids (Benjamin, New York, 1964).

${ }^{39}$ F. A. Lindemann, Phys. Z. 11, 609 (1910).

${ }^{40}$ G. C. Abell, Phys. Rev. B 31, 6184 (1985).

${ }^{41}$ J. Tersoff, Phys. Rev. Lett. 56, 632 (1986).

${ }^{42}$ N. R. Comins, Philos. Mag. 25, 817 (1972).

${ }^{43}$ M. Kandyla, T. Shih, and E. Mazur, Phys. Rev. B 75, 214107 (2007).

${ }^{44}$ H. Iglev, M. Schmeisser, K. Simeonidis, A. Thaller, and A. Laubereau, Nature (London) 439, 183 (2006).

${ }^{45}$ O. P. Uteza, E. G. Gamaly, A. V. Rode, M. Samoc, and B. Luther-Davies, Phys. Rev. B 70, 054108 (2004).

${ }^{46}$ B. J. Siwick, J. R. Dwyer, R. E. Jordan, and R. J. D. Miller, Science 302, 1382 (2003). 